\title{
Intelligent computing in science and technology
}

\author{
Jose Antonio Marmolejo-Saucedo ${ }^{1} \cdot$ Pandian Vasant $^{2}$
}

Published online: 29 June 2020

(C) Springer Science+Business Media, LLC, part of Springer Nature 2020

\section{Contents}

Binary monkey algorithm for approximate packing noncongruent circles in a rectangular container

Rafael Torres-Escobar, José Antonio Marmolejo-Saucedo and Igor Litvinchev

Nature-inspired meta-heuristics approaches for charging plug-in hybrid electric vehicle

Pandian Vasant, Jose Antonio Marmolejo, Igor Litvinchev and Roman Rodriguez Aguilar

Vertical and horizontal integration systems in Industry 4.0

Magdiel Pérez-Lara, Jania Astrid Saucedo-Martínez, José Antonio Marmolejo-Saucedo, Tomás Eloy SalaisFierro and Pandian Vasant

Optimization of public resources through an ensemblelearning model to measure quality perception in the social protection system in health of Mexico

Roman Rodriguez-Aguilar and Gustavo Rivera-Peña

Better campus life for visually impaired University students: intelligent social walking system with beacon and assistive technologies

Utku Kose and Pandian Vasant

Effect of ICT integration on SC flexibility, agility and company' performance: the Mexican maquiladora experience

Jorge Luis García-Alcaraz, Valeria Martínez-Loya, José Roberto Díaz-Reza, Julio Blanco-Fernández, Emilio Jiménez-Macías and Alfonso Jesús Gil López.

Jose Antonio Marmolejo-Saucedo

jmarmolejo@up.edu.mx

1 Facultad de Ingenieria, Universidad Panamericana, Augusto Rodin 498, 03920 Ciudad de Mexico, Mexico

2 University Technology Petronas, 31750 Tronoh, Malaysia

\section{Editorial}

The requirements demand in problem solving have been increasingly in demand in exponential way. The new technologies in computer science and engineering have reduced the dimension of data coverage worldwide. Thus, the recent inventions in Information and Communications Technologies have inched towards reducing the gaps, and coverage of domains globally. The digging of information in a large data, and the soft-computing techniques have contributed a strength in prediction, analysis, and decision potentials in the niche areas such as Computer Science, Technology, Management, Social Computing, Green Computing, and Telecom. Nurturing the research in Technology and Computing are evident that finding a right pattern in the ocean of data.

Additionally, classic optimization problems become even more complex in today's organizational environment. The topics of industry 4.0, logistics, social problems solved by optimization and the classic challenges in the manufacturing industry continue to be a challenge for new developments and research.

This special issue features six selected papers with high quality. The first article, "Binary monkey algorithm for approximate packing non-congruent circles in a rectangular container", authored by Rafael Torres-Escobar, José Antonio Marmolejo-Saucedo and Igor Litvinchev, proposed the packing problem for non-congruent (different size) circles with the binary version of the monkey algorithm which incorporates a cooperation process and a greedy strategy. The authors use a rectangular grid for covering the container. Every node in the grid represent potential positions for a circle. In this sense, binary monkey algorithm for the knapsack problem, can be used to solve de $0-1$ approximate packing problem for non-congruent circles. The binary monkey problem uses two additional processes of the original monkey algorithm, these two processes are a greedy process and a cooperation process.

The second article titled "Nature-inspired meta-heuristics approaches for charging plug-in hybrid electric vehicle" present four swarm intelligence based optimization 
techniques: particle swarm optimization (PSO), gravitational search algorithm (GSA), accelerated particle swarm optimization, and hybrid version of PSO and GSA (PSOGSA) have been applied for the state-of-charge optimization of plug-in hybrid electric vehicles. In this research, hybrid PSOGSA has performed very well in producing better results than other stand-alone optimization techniques.

In the next article with the title "Vertical and horizontal integration systems in Industry 4.0", Perez-Lara et al. propose and validate a methodological tool, for evaluating the technological and operational criteria within companies and place them in the right level for a transfer to the new industrial revolution, considering as well the vertical and horizontal systems in Industry 4.0. The evaluation tool will result in current vertical and horizontal level company integration. The study allows identifying gaps and opportunities to develop other blocks considered in Industry 4.0

The fourth article titled "Optimization of public resources through an ensemble-learning model to measure quality perception in the social protection system in health of Mexico" consider a model of ensemble learning to measure the perception of quality in the medical care granted to the people affiliated to the social protection in health system of Mexico. The study allows a more efficient allocation of resources based on the main areas of opportunity identified in the measurement of service quality. A satisfaction index was built using an ensemble model using principal component analysis, logistic model and bagging meta-estimator, to identify the effect of a group of factors in the perception of quality of health services and monitor the perceived quality of users in real time.

The fifth article, "Better campus life for visually impaired University students: intelligent social walking system with beacon and assistive technologies" proposed a introduce a novel, low-cost intelligent social walking path support system for visually impaired students in a wide campus area, by employing beacons, optimization based Artificial Intelligence techniques, Big Data support, and a system rising over Internet of Things. In detail, the developed system has been used within two connected campus areas of Suleyman Demirel University located in the city of Isparta in Turkey and an effective walking path support was ensured for enabling visually students to go target locations with instructions given by an intelligent system.

The last article titled "Effect of ICT integration on SC flexibility, agility and company' performance: the Mexican maquiladora experience" reports a structural equation model (SEM) with four latent variables to measure the relationship between information and communication technologies (ICT) integration with supply chain flexibility, supply chain agility, and company's performance. The
SEM integrates six hypotheses with relationships among variables and is validated with 378 responses from manufacturing sector to a questionnaire and partial least squares technique is used to evaluate it and test the hypotheses statistically. A sensitivity analysis is conducted in different scenarios to know conditional probabilities of occurrence of dependent variables, since a scenario has occurred in the independent variable with low and high success level.

Acknowledgement The editors appreciate the effort and work of the reviewers to enrich the quality of this special issue. We also thank the Edit-in-Chief, Dr. Imrich Chlamtac for his supportive guidance during the entire process.

Publisher's Note Springer Nature remains neutral with regard to jurisdictional claims in published maps and institutional affiliations.

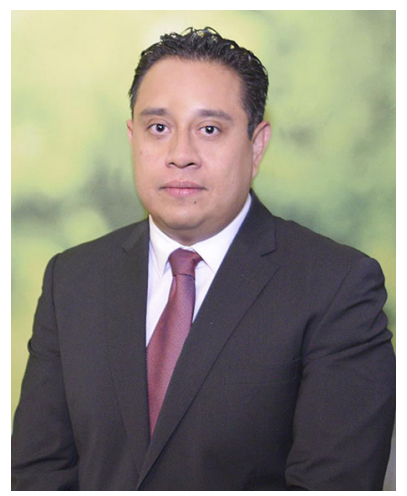

Jose Antonio Marmolejo-Saucedo is a Professor at Panamerican University, Mexico. His research is on operations research, design and development of digital twins, largescale optimization techniques, computational techniques and analytical methods for planning, operations, and control of electric energy and logistic systems. $\mathrm{He}$ is particularly interested in topics related to artificial intelligence, digital twins, the Internet of things and Industry 4.0. He received his Doctorate in Operations Research (Hons) at National Autonomous University of Mexico. At present, He has the second highest country-wide distinction granted by the Mexican National System of Research Scientists for scientific merit (SNI Fellow, Level 2). He is a member of the Network for Decision Support and Intelligent Optimization of Complex and Large Scale Systems and Mexican Society for Operations Research. He has co-authored research articles in science citation index journals, conference proceedings, presentations, books, and book chapters.

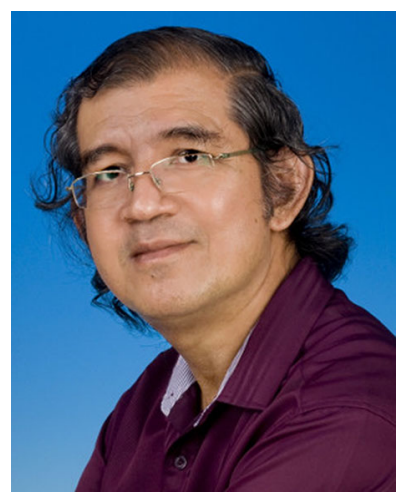

Pandian Vasant is a senior lecturer at Department of Fundamental and Applied Sciences, Faculty of Science and Information Technology, Universiti Teknologi PETRONAS in Malaysia. He holds Ph.D. (UNEM, Costa Rica) in Computational Intelligence, M.Sc. (UMS, Malaysia, Engineering Mathematics) and B.Sc. (Hons, Malayan University (MU), Malaysia) in Mathematics. His research interests include Soft Computing, Hybrid Optimization, Holistic Optimization, Innovative Computing and Applications. $\mathrm{He}$ has co-authored research papers and articles in national journals, international journals, conference proceedings, conference paper presentation, and special issues lead guest editor, lead guest editor for book chapters' project, conference abstracts, edited books, keynote lecture and book chapters (161 publications indexed in SCOPUS). In 
the year 2009, Dr. Pandian Vasant was awarded top reviewer for the journal Applied Soft Computing (Elsevier) and awarded outstanding reviewer in the year 2015 for ASOC (Elsevier) journal. He has 25 years of working experience at the various universities from 1989 to 2016. Currently he is Editor-in-Chief of IJCO, IJSIEC, IEM, IJEOE and Editor of GJTO. H-Index SCOPUS Citations = 35, H-Index Google Scholar $=22$. 